\title{
ANAPHYLACTIC REACTION TO PATENT BLUE DYE DURING SENTINEL LYMPH NODE BIOPSY IN BREAST CANCER
}

\author{
Flavio Rocha Gil'1, Mariane de Melo Silveira', Giovana Vilela Rocha', Plinio Resende de Melo Filho \\ ${ }^{1}$ Centro Universitário de Patos de Minas - Patos de Minas (MG), Brazil.
}

Introduction: Sentinel lymph node biopsy is indicated as gold standard in the surgical treatment of initial breast cancer, presenting as a more conservative approach and preventing total lymphadenectomy. Dyes or technetium radiopharmaceuticals can be used to identify the sentinel lymph node. The most used dyes for the identification of the sentinel lymph node are patent blue and isosulfan blue, and, in lower frequency, methylene blue. However, hypersensitivity reactions to blue dye have been described, estimating its prevalence in $0.6 \%-2.7 \%$. The clinical status that characterizes the allergic reaction to the dye can range from mild skin changes to the severe condition, with circulatory collapse. Objectives: To present a severe case of allergic reaction to patent blue in a patient submitted to surgical treatment for breast cancer. Method: This is a case report study based on the analysis of medical records and literature review. Case report: T.L.O.M, female, 49-year old woman, white, married, born in Patos de Minas, MG. Patient diagnosed with Breast cancer, T1N0A0, with indication for sentinel lymph node analysis with blue patent and segmental resection of the tumor. ASA 1 pre-anesthetic evaluation, without history of allergy. The patient was submitted to general inhalation anesthesia and subcutaneous injection in the left breast of $2 \mathrm{ml}$ of patent blue, followed by massage. During the anesthetic plan, after 40 minutes of surgery, the patient was hypotensive (40x20 mmHg), with low saturation (ETCO2 28), tachycardia (120 bpm), associated with bluish urticariform papules, and major edema in the ear lobes, being immediately assisted with adrenaline bolus, metaraminol, and decadron. The patient became stable, and it was possible to conclude the procedure. Then, she was referred to the intensive care unit, under sedation and intubated due to the risk of laryngospasm. She evolved hemodynamically stable, and was extubated without intercurrences. Conclusions: A severe anaphylactic reaction to patent blue can risk the life of a patient in an unpredictable manner. Considering this case, it is essential that the entire staff involved in the sentinel lymph node biopsy be aware of the possibility of a hypersensitive reaction to the dye, being prepared to recognize and immediately handle the possible repercussions. 